\title{
Discussion on the Effectiveness of Elevating HDL-C in Treating Cardiovascular Diseases of Patients with Type 2 Diabetes Mellitus
}

\author{
Shasha Wang1, Liyue Wang² \\ ${ }^{1}$ Medical College, Wuhan University of Science and Technology, Wuhan, China \\ ${ }^{2}$ Puren Hospital Affiliated to Wuhan University of Science and Technology, Internal Medicine-Cardiovascular \\ Department, Wuhan, China \\ Email:247541284@qq.com
}

How to cite this paper: Wang, S.S. and Wang, L.Y. (2019) Discussion on the Effectiveness of Elevating HDL-C in Treating Cardiovascular Diseases of Patients with Type 2 Diabetes Mellitus. International Journal of Clinical Medicine, 10, 293-305. https://doi.org/10.4236/ijcm.2019.104022

Received: March 2, 2019

Accepted: April 13, 2019

Published: April 16, 2019

Copyright $\odot 2019$ by author(s) and Scientific Research Publishing Inc. This work is licensed under the Creative Commons Attribution International License (CC BY 4.0).

http://creativecommons.org/licenses/by/4.0/

\begin{abstract}
High density lipoprotein protects cardiovascular diseases and reduces the risk of cardiovascular diseases through cholesterol reverse transport and other mechanisms. High-density lipoprotein cholesterol (HDL-C) is an independent predictor of negative events in cardiovascular diseases. Low concentration of HDL-C indicates abnormal regulation of HDL anabolism. Various proteins and receptors such as cholesteryl ester transfer protein (CETP) are involved in HDL anabolism. Type 2 Diabetes Mullitu and its related metabolic syndrome, chronic inflammation as well as oxidative stress not only affect the proteins and receptors related to HDL anabolism, but also affect their functional changes, making HDL change from anti-inflammatory, antioxidant, protecting endothelial cell function to pro-inflammatory, pro-oxidative and pro-endothelial cell apoptosis. This article will describe the relationship between HDL-C, type 2 diabetes and cardiovascular diseases from the effects of T2DM on HDL anabolism and function, and further explore the effectiveness of elevating HDL-C in treating cardiovascular diseases of patients with type 2 diabetes.
\end{abstract}

\section{Keywords}

HDL, Type 2 Diabetes Mellitus, Cardiovascular Diseases, Reverse Cholesterol Transport

\section{Introduction}

Patients with diabetes mellitus are high-risk groups for cardiovascular and cerebrovascular diseases. The risk of cardiovascular and cerebrovascular diseases in 
patients with diabetes mellitus is 2 - 4 times higher than that of non-patients with diabetes mellitus. Compared with non-patients with diabetes mellitus with coronary heart disease, patients with diabetes mellitus with coronary heart disease account for $67 \%$ of coronary heart disease-related deaths. About $84 \%$ of patients with diabetes mellitus aged over 65 die from cardiovascular and cerebrovascular events [1]. Therefore, diabetes patients, a large group of people, have attracted extensive attention. Both type 2 diabetes mellitus and its metabolic syndrome can affect HDL [2]. Moreover, chronic inflammation, oxidative stress and other factors of diabetes can lead to changes in HDL serum concentration, structure and function, and even change from functional HDL with anti-inflammation, anti-oxidation, anti-apoptosis and anti-thrombosis to dysfunction HDL with pro-inflammation, pro-oxidation, pro-apoptosis and pro-thrombosis functions, thus increasing the risk of cardiovascular diseases. As a result, the effectiveness of elevating HDL-C as a therapeutic target to reduce the risk of cardiovascular diseases in patients with type 2 diabetes mellitus has been widely questioned. This article will review the effectiveness of elevating HDL-C in treating cardiovascular diseases in patients with type 2 diabetes mellitus from the aspects of the effects of type 2 diabetes mellitus on HDL anabolism and function.

\section{HDL}

\subsection{Structure}

In plasma, HDL is the lipoprotein with the largest density and the smallest volume in human body (Density: $1.063-1.21 \mathrm{~g} / \mathrm{ml}$ ) [3]. The main protein component of HDL is apolipoprotein A-I(apo A-I), accounting for about 70\%. Therefore, apo A-I is closely related to HDL-C concentration. Proteomics research continues to expand apolipoprotein spectrum and other related protein components in HDL [4]. HDL lipids are mainly free or esterified fatty acids, phospholipids and different ceramides and sphingolipids [5]. Unlike other apolipoproteins, HDL exists as apolipoprotein precursor particles rather than mature apolipoproteins. These particles are mostly disk-shaped and consist of phospholipid bilayers and apolipoprotein stabilized unesterified cholesterol [6]. There are many subgroups of HDL, which are classified by volume: HDL3c, HDL3b, HDL3a, HDL2a, HDL2b; According to density, there are HDL2 ( $d=1.063$ $1.125 \mathrm{~g} / \mathrm{ml})$, HDL3 ( $\mathrm{d}=1.125-1.21 \mathrm{~g} / \mathrm{ml})$, etc. [7].

\subsection{Synthesis and Decomposition of HDL and Its Function-Related Proteins}

The HDL subgroups increase or eliminate the neutral lipids, phospholipids in the components under the reaction of related proteins to undergo reconstruction or transformation, and proteins involved in HDL synthesis and decomposition and function-related include: 


\subsubsection{Serum Amyloid A (SAA)}

SAA competitively combines with apo A-I and HDL, and can become the main protein component of HDL. HDL containing SAA mediates the decrease of cholesterol outflow activity [8] and is easier to be cleared by kidney. It has been reported that SAA has a certain role in the development of plaque.

\subsubsection{CETP}

CETP mediates the exchange of cholesteryl esters in HDL with triglyceride-rich lipoproteins to promote the reverse transport of cholesterol, so that the increase of TG component in HDL increases the catabolism and leads to a decrease in HDL-C concentration.

\subsubsection{Endothelial Lipase (EL)}

EL is consistent with liver lipase and lipoproteinase, and tends to hydrolyze triglyceride-rich lipoproteins. Current experimental studies have shown that overexpression of adenovirus gene into EL reduces the cholesterol component and phospholipid component of HDL.

\subsubsection{Lecithin: Cholesterol Acytransferase (LCAT)}

LCAT needs to transfer sn-2-acyl of lecithin to cholesterol under the action of apo A-I co-activation factor, so as to convert free cholesterol in HDL into cholesteryl ester. This process retains the core of HDL particles, and converts naive HDL into spherical particles, which is especially important for reverse cholesterol transport process.

\subsubsection{Paraoxonase (PON)}

PON1 is an enzyme related to HDL which can protect LDL from oxidation as well as an important mechanism for HDL to resist atherosclerosis. The reduction of PON1 can reduce HDL antioxidant function [9]. Moreover, HDL-PON can decompose peroxides on the surface of cell membrane. Some researchers have found that there is a negative correlation between HDL-PON activity and HDL peroxide level, which proves that PON not only reduces LDL and membrane oxidation, but also reduces HDL oxidation.

\subsubsection{ATP-Binding Cassette Transporter A1 and G1 (ABCA1/ABCG1)}

ABCA1 plays an important role in reverse cholesterol transport and HDL synthesis. ABCA1 promotes cholesterol efflux process (at least lipid apo A-I from cells) by consuming ATP to participate in cholesterol reverse transport process. The role of ABCG1 is to re-esterify apo A-I produced by ABCA1 pathway through energy consumption mediated lipid outflow.

\subsection{HDL-Cholesterol Reverse Transport}

Apo A-I, the skeleton of HDL protein, is mainly synthesized and secreted into circulation by liver and intestinal tract, and combines free phospholipids, cholesterol and triglycerides to form juvenile HDL particles [10]. Juvenile HDL is bound to the surface of peripheral tissues such as macrophages ABCA1 activates 
intracellular cholesterol ester hydrolase to produce free cholesterol, which is transported to juvenile HDL [11], and forms cholesterol ester after LCAT catalysis, making it easier to embed into HDL core. This process converts low-fat HDL precursor particles into lipid-rich HDL (mature HDL) [12]. Subsequent mature HDL is released into the circulation and reaches the liver in combination with scavenger receptor-B1 (SR-B1), making HDL lipid components more susceptible to hydrolysis by lipases such as hepatic lipase to unload their lipid components [13]. Subsequently, lipid-poor HDL is released into the circulation to repeat the above-mentioned cholesterol transport process [14], which is the reverse transport of cholesterol-HDL removes excess cholesterol from the periphery and transports it to the liver for metabolism or to steroid synthesis organs for steroid hormonessynthesis. Thus, HDL prevents lipid toxicity and reduces the formation of foam cells, thereby reducing the formation of atherosclerotic plaque and reducing the risk of cardiovascular events [15].

\section{HDL, Diabetes Mellitus and Cardiovascular Diseases}

Cardiovascular diseases are still the leading cause of death in many developed and developing countries [12]. HDL's reverse cholesterol transport, anti-inflammatory [16], antioxidant and other functions play a great role in cardiovascular protection [17]. Type 2 diabetes mellitus and its metabolic syndrome affect HDL concentration and function in many ways.

\subsection{Changes in Anabolism and Catabolism}

Changes in anabolism and catabolism of HDL are accompanying insulin resistance, resulting in HDL synthesis with little triglyceride and little cholesterol ester, non-enzymatic saccharification of apo A-I and other HDL related proteins, oxidative modification of HDL lipids, apolipoproteins and enzymes, etc. [18].

\subsubsection{Proteomic Changes}

Chronic inflammation in patients with type 2 diabetes mellitus increases SAA [19]. SAA replaces apo A-I from HDL surface, and SAA-rich HDL is more susceptible to hydrolytic metabolism. Oxidative stress in patients with diabetes mellitus is increasing, resulting in a decrease in HDL-related proteins apo A-I and PON1.

\subsubsection{Lipid Histological Changes}

Insulin resistance and T2DM can increase the level of TG. Hypertriglyceridemia increases CETP-mediated transesterification of TG-rich lipoprotein and HDL cholesterol, which increases the content of TG-rich HDL (poor stability and loose binding with Apo-A1) in circulation and accelerates renal excretion [20]. Phospholipids in T2DM have changed, and the ratio of sphingomyelin/lecithin (the main determinant of HDL antioxidant function) has increased.

\subsection{Functional Changes}

The function of HDL depends on the shape of HDL (spherical shape is better 
than discoid shape [21]), the size and composition of HDL (fatty acid composition/triglyceride content [22], glycated protein, peroxide protein).

\subsubsection{Impaired Reverse Cholesterol Transport Function}

$A B C A 1$ and $A B C G 1$ are key components in cholesterol reverse transport. Their expression in macrophages up-regulates lipid loading [23], while ABCA1 mediates cholesterol and phospholipid efflux to apo A-I, and phospholipids and cholesterol in mature HDL are mainly derived from ABCG1-mediated lipid efflux [24]. Mauldin et al. [25] demonstrated that hyperglycemia resulted in decreased ABCG1 expression and impaired function of macrophages in a mouse model of type 2 diabetes mellitus. In vitro experiments, RAW264.7 macrophages and HepG2 hepatocytes were incubated in unsaturated fatty acid medium, and the expression of ABCA1 mRNA and protein were both down-regulated [26]. Significantly elevated unsaturated fatty acids in patients with diabetes mellitus have been shown to phosphorylate ABCA1 through phospholipase D2 pathway and reduce its stability, thus affecting its function [27] and reducing cholesterol reverse transport. The increased SAA in T2DM patients binds to HDL and increases its affinity to macrophages by 3 to 4 times, while the affinity to hepatocytes decreases. The difference in affinity may lead to HDL switching from liver to macrophage clearance, which may be closely related to HDL's anti-atherosclerosis and anti-inflammatory properties changing to atherosclerosis promoting properties [8]. In addition, SAA has been shown to reduce HDL subpopulations and promote cholesterol outflow [28]. Advanced glycation products (AGEs) formed in patients with diabetes mellitus are increased, and AGE precursors hinder ABCA1-dependent intracellular cholesterol efflux [29]. In addition, AGEs [30] were also detected from lipoprotein isolated from diabetic subjects. The increase in apo A-I carbonyl modification associated with AGEs [30] may be related to abnormal HDL metabolism. A recent study shows that inflammation destroys many components of HDL cholesterol transport and efflux function [31]. Inflammation-induced down regulation of bile transporters ABCG5, ABCG8 and ABCB11 in the liver results in impaired cholesterol metabolism through the liver. The above-mentioned various mechanisms damage the reverse cholesterol transport process.

\subsubsection{Impaired Antioxidant Function}

Chronic inflammation induces the secretion of myeloperoxidase (MPO), and MPO activity increases in HDL in patients with type 2 diabetes mellitus [32]. MPO has been proved to selectively catalyzenitrosation, chlorination, oxidation of Apo-A1, and oxidized apo A-I selectively inhibit ABCA1-dependent cholesterol outflow process [33]. Apo A-I has a pro-inflammatory effect after oxidation by MPO. Oxidative modification of HDL apolipoproteins and enzymes in patients with diabetes [22] and increased sphingomyelin/phosphatidylcholine ratios lead to an increase in the surface hardness of HDL (a key antioxidant factor [34]), which reduces their antioxidant activity. The content of very low density 
lipoprotein increases in type 2 diabetes mellitus, which is proved to be an effective LCAT activity inhibitor [35]. In addition to its importance in reverse cholesterol transport, LCAT can hydrolyze platelet activating factor (PAF) [36] [37] and phosphatidylcholine produced during lipoprotein oxidation, eliminating its pro-inflammatory function and cytotoxicity. In addition to its antioxidant properties, LCAT has an effect on the concentration of PON1 and PAF acetylhydrolase [38]. This indicates that LCAT plays an important role in the reverse cholesterol transport and HDL antioxidant function.

The anti-atherosclerosis effect of HDL is closely related to the regulation of LDL and cell membrane oxidation by PON1. Increased oxidative stress in T2DM reduces the concentration of PON1 [39], which is $40 \%$ of that in non-patients with diabetes mellitus [40]. PON1 inhibits the production of monocyte chemotactic protein 1 (MCP1) mediated by oxLDL through endothelial cells [41]. MCP1 induces monocytes to recruit subcutaneously, which lays a foundation for the occurrence of atherosclerosis. The protective effect of PON1 transfected cells and PON1 transgenic mice on macrophage oxidative stress [42] reduces the formation of atherosclerotic lesions [43], and PON1 deficiency is found to be related to macrophage oxidative stress and increased atherosclerosis [44]. The more peroxide components in HDL, the lower HDL-PON activity [45]. In short, the decrease of PON activity and HDL anti-membrane and LDL oxidation protection in patients with diabetes mellitus accelerates atherosclerosis in patients with diabetes mellitus.

\subsubsection{Impaired Endothelial Protective Function}

Some studies have found that the protective effect of HDL on vascular endothelium is obviously impaired in patients with T2DM [32]. Perségol et al. [46] proved that HDL lipid peroxidation and myeloperoxidase (MPO) activity increased in patients with $\mathrm{T} 2 \mathrm{DM}$, and the ability of resisting oxidized low density lipoprotein to inhibit endothelium-dependent vasodilation was impaired. Increased MPO can increases endothelial dysfunction [47] [48] and coronary heart disease risk [49]. In vitro studies have shown that HDL isolated from healthy individuals incubates endothelial cells and found that HDL could induce the expression of endothelial NO synthase (eNOS). However, the loss of HDL vasodilation function in patients with T2DM is due to the reduction of NO production and endothelium-dependent relaxation function. Moreover, in patients with diabetes and coronary heart disease, HDL's function of inhibiting expression of endothelial intercellular adhesion molecule-1 (VCAM-1) is absent, thus macrophages are more likely to adhere to activated vascular endothelial cells [47], and endothelium is more likely to be damaged. Recent studies have shown that HDL endothelial protective function mainly increases the number of serum endothelial progenitor cells (EPCs) [50] and accelerates endothelial cell neogenesis. Some studies have found that HDL loss from T2DM patients with coronary heart disease inhibits endothelial cell apoptosis because it cannot activate anti-apoptotic proteins and stimulate the pro-apoptotic pathway. In vitro, cardi- 
omyocytes are incubated with high glucose, and HDL supplementation can offset the apoptosis-promoting effect of some high glucose on cardiomyocytes. In healthy control group and patients with diabetes mellitus, low HDL is an independent risk factor for endothelial dysfunction [51].

\section{Diabetes Mellitus and Coronary Heart Disease}

Numerous epidemiological studies have shown that diabetes is one of the independent risk factors for cardiovascular diseases. Endothelial dysfunction plays an important role in the pathogenesis of microangiopathy in patients with diabetes mellitus [52], and is the main cause of diabetes morbidity and mortality. At the early stage of atherosclerosis formation, endothelial dysfunction already exists, while there is no obvious morphological change in vascular wall at this time. Endothelial dysfunction is common in patients with diabetes mellitus. eNOS expression is down-regulated, NO synthesis is reduced, and endothelium-dependent vasodilation is abnormal [53]. Diabetes-related hyperglycemia and increase of angiotensin II (Ang II) can increase reactive oxygen species (ROS) in circulation. Oxidative stress causes endothelial dysfunction [54], partly due to NO degradation. NO has important antithrombotic, anti-apoptosis and anti-inflammatory functions. This makes people wonder whether other functions such as anti-inflammatory function and anti-apoptosis function are also changed after vascular endothelial relaxation dysfunction. A large number of studies have shown that activation of angiotensin II type 1 receptor (AT1R) increases vascular endothelial oxidation products and initiates apoptosis process, which promotes the occurrence and development of vascular endothelial dysfunction [54]. The expression level of AT1R determines its biological activity. Currently, several known agonists include glucose, angiotensin II, insulin, reactive oxygen species and others including diabetes itself [53]. The understanding of up-regulation of AT1R expression and angiotensin II mediated signaling in patients with diabetes mellitus comes from the discovery that endothelial function is repaired after patients with diabetes mellitus use AT1R antagonist [55]. Some studies have found that endothelial progenitor cells (EPCs) are also targeted to repair damaged sites after vascular wall integrity is destroyed, in addition to repairing adjacent mature endothelial cells [56]. EPC is not only reduced in number but also impaired in function in patients with diabetes mellitus [57], which may be related to endothelial dysfunction [58]. Primary myocardial injury in patients with diabetic heart disease occurs before hypertension and coronary artery disease. Ventricular dysfunction in diabetic heart disease is mainly due to myocardial cell apoptosis, interstitial inflammatory reaction, myocardial cell hypertrophy, glycogen accumulation in myocardium, changes in myocardial extracellular matrix (interstitial and perivascular fibrosis), myocardial microvascular lesions, intracellular $\mathrm{Ca}^{2+}$ abnormalities and endothelial cell dysfunction [59]. Some animal studies have found that cell apoptosis increases in diabetic animals induced by streptozotocin (STZ) [60], as well as in patients with di- 
abetes mellitus [61]. Apoptosis is mainly due to increased oxidative stress caused by hyperglycemia. Hyperglycaemia not only increases the production of reactive oxygen species, but also inhibits the production of antioxidant enzymes [62]. In addition to inducing lipid peroxidation, ROS can also change cell proteins and initiate various stress signal pathways, such as Erk, JNK and p38 MAPK. Activation of cardiac p38 MAPK is of pathological importance in diabetic heart disease, which indicates that inhibition of p38 MAPK can improve STZ-induced left ventricular dysfunction in diabetic mice [63]. It has been confirmed in transgenic and knockout animal models that antioxidant enzymes superoxide dismutase (SOD), SOD-1, SOD-2, and extracellular (ec)-SOD convert $\mathrm{O}_{2}$-anions into oxygen molecules and hydrogen peroxide [64], and SOD-2 is the same in patients with diabetes mellitus [65]. In the heart, overexpression of ec-SOD reduces macrophage infiltration and fibrosis, and improves left ventricular dysfunction [64], while up-regulation of SOD-2 expression can prevent apoptosis induction and protect mitochondrial respiratory function [66].

\section{Conclusion}

To sum up, HDL has long been considered as a protective component of cardiovascular diseases, and HDL-C concentration is a good predictor of cardiovascular disease risk factors. Type 2 diabetes mellitus affects HDL metabolism and thus reduces HDL-C concentration, but increasing HDL-C concentration with drugs has not been able to reduce the risk of cardiovascular events as scheduled. Not only that, T2DM changes HDL in size, shape and composition, thus changing its function and even transforming it from cardiovascular protective effects such as anti-inflammation, anti-apoptosis and antithrombotic to cardiovascular disease events such as pro-inflammation, pro-apoptosis and thrombosis. Therefore, we should not only pay attention to HDL-C concentration, but also pay attention to its morphology, composition and functional changes in the treatment of type 2 diabetes patients with cardiovascular diseases, which provides a new research direction for cardiovascular disease risk prediction and treatment targeting of patients with T2DM.

\section{Conflicts of Interest}

The authors declare no conflicts of interest regarding the publication of this paper.

\section{References}

[1] Kunutsor, S.K., Kieneker, L.M., Bakker, S.J.L., James, R.W. and Dullaart, R.P.F. (2017) Incident Type 2 Diabetes Is Associated with HDL, But Not with Its Anti-Oxidant Constituent-Paraoxonase-1: The Prospective Cohort PREVEND Study. Metabolism, 73, 43-51. https://doi.org/10.1016/j.metabol.2017.05.004

[2] Yang, S.H., Du, Y. and Li, X.L. (2017) Triglyceride to High-Density Lipoprotein Cholesterol Ratio and Cardiovascular Events in Diabetics with Coronary Artery Disease. The American Journal of the Medical Sciences, 354, 117-124.

https://doi.org/10.1016/j.amjms.2017.03.032 
[3] Chang, T.I., Streja, E. and Moradi, H. (2017) Could High-Density Lipoprotein Cholesterol Predict Increased Cardiovascular Risk. Current Opinion in Endocrinology, Diabetes and Obesity, 24, 140-147. https://doi.org/10.1097/MED.0000000000000318

[4] Hermans, M.P., Valensi, P. and Ahn, S.A. (2018) [HDL-C/apoA-I]: A Multi-Vessel Cardiometabolic Risk Marker in Women with T2DM. Diabetes/Metabolism Research and Reviews, 34, 829-840. https://doi.org/10.1002/dmrr.2950

[5] Wiesner, P., Leidl, K., Boettcher, A., Schmitz, G. and Liebisch, G. (2009) Lipidprofiling of FPLC-Separated Lipoprotein Fractions by Electrosprayionization Tandem Mass Spectrometry. The Journal of Lipid Research, 50, 574-585. https://doi.org/10.1194/jlr.D800028-JLR200

[6] Van Linthout, S., Spillmann, F., Schultheiss, H.P. and Tschöpe, C. (2010) High-Density Lipoprotein at the Interface of Type 2 Diabetes Mullitus and Cardiovascular Disorders. Current Pharmaceutical Design, 16, 1504-1516.

[7] Park, K.H., Shin, D.G. and Cho, K.H. (2014) Dysfunctional Lipoproteins from Young Smokers Exacerbate Cellular Senescence and Atherogenesis with Smaller Partical Size and Severe Oxidation and Glycation. Toxicological Sciences, 140, 16-25. https://doi.org/10.1093/toxsci/kfu076

[8] Shao, B., Tang, C., Sinha, A., Mayer, P.S., Davenport, G.D., Brot, N., et al. (2014) Humans with Atherosclerosis Have Impaired ABCA1 Cholesterol Efflux and Enhanced High-Density Lipoprotein Oxidation by Myeloperoxidase. Circulation Research, 114, 1733-1742. https://doi.org/10.1161/CIRCRESAHA.114.303454

[9] Mackness, M. and Mackness, B. (2015) Human Paraoxonase-1 (PON1): Gene Structure and Expression, Promiscuous Activities and Multiple Physiological Roles. Gene, 567, 12-21. https://doi.org/10.1016/j.gene.2015.04.088

[10] Rosenson, R.S., Brewer, H.B., Davidson, W.S., Fayad, Z.A., Fuster, V., Goldstein, J., et al. (2012) Cholesterol Efflux and Atheroprotection: Advancing the Concept of Reverse Cholesterol Transport. Circulation, 125, 1905-1919. https://doi.org/10.1161/CIRCULATIONAHA.111.066589

[11] Wang, X., Collins, H.L., Ranalletta, M., Fuki, I.V., Billheimer, J.T., et al. (2007) Macrophage ABCA1 and ABCG1, But Not SR-BI, Promote Macrophage Reverse Cholesterol Transport in Vivo. Journal of Clinical Investigation, 117, 2216-2224. https://doi.org/10.1172/JCI32057

[12] Rosamond, W., Flegal, K., Friday, G., et al. (2007) Heart Disease and Stroke Statistics-2007 Update: A Report from the American Heart Association Statistics Committee and Stroke Statistics Subcommittee. Circulation, 115, 69-71.

[13] Oda, M.N. (2015) High-Density Lipoprotein Cholesterol: Origins and the Path Ahead. Current Opinion in Endocrinology, Diabetes and Obesity, 22, 133-141. https://doi.org/10.1097/MED.0000000000000139

[14] Hirschler, V., Maccallini, G., Sanchez, M., Gonzalez, C. and Molinari, C. (2015) Association between Triglyceride to HDL-C Ratio and Insulin Resistance in Indigenous Argentinean Children. Pediatric Diabetes, 16, 606-612. https://doi.org/10.1111/pedi.12228

[15] Rosenson, R.S., Brewer, H.B., Ansell, B.J., et al. (2016) Dysfunctional HDL and Atherosclerotic Cardiovascular Disease. Nature Reviews Cardiology, 13, 48-60. https://doi.org/10.1038/nrcardio.2015.124

[16] Rosenson, R.S., Brewer Jr., H.B., Ansell, B., et al. (2013) Translation of High-Density Lipoprotein Function into Clinical Practice: Current Prospects and Future Challenges. Circulation, 128, 1256-1267. 
https://doi.org/10.1161/CIRCULATIONAHA.113.000962

[17] AIM-HIGH Investigators, Boden, W.E., Probstfield, J.L., et al. (2011) Niacin in Patients with Low HDL Cholesterol Levels Receiving Intensive Stat in Therapy. The New England Journal of Medicine, 367, 2255-2267. https://doi.org/10.1056/NEJMoa1107579

[18] Voight, B.F., Peloso, G.M., Orho-Melander, M., et al. (2012) Plasma HDL Cholesterol and Risk of Myocardial Infarction: A Mendelian Randomisation Study. The Lancet, 380, 572-580. https://doi.org/10.1016/S0140-6736(12)60312-2

[19] Huang, M.C., Chang, W.T., Chang, H.Y., et al. (2017) FADS Gene Polymorphisms, Fatty Acid Desaturase Activities, and HDL-C in Type 2 Diabetes. International Journal of Environmental Research and Public Health, 14, pii: E572.

[20] Pérez-Méndez, Ó., Pacheco, H.G., Martínez-Sánchez, C., et al. (2014) HDL-Cholesterol in Coronary Artery Disease Risk: Function or Structure? Clinica Chimica Acta, 429, 111-122. https://doi.org/10.1016/j.cca.2013.12.001

[21] Song, X., Teng, J., Wang, A., et al. (2016) Positive Correlation between Serum IGF-1 and HDL-C in Type 2 Diabetes Mellitus. Diabetes Research and Clinical Practice, 118, 44-49. https://doi.org/10.1016/j.diabres.2016.04.056

[22] Patel, S., Puranik, R., Nakhla, S., et al. (2009) Acute Hypertriglyceridaemia in Humans Increases the Triglyceride Content and Decreases the Anti-Inflammatory Capacity of High Density Lipoproteins. Atherosclerosis, 204, 424-428. https://doi.org/10.1016/j.atherosclerosis.2008.07.047

[23] Zhang, L., Chen, S., Deng, A., Liu, X., Liang, Y., Shao, X., et al. (2015) Association between Lipid Ratios and Insulin Resistance in a Chinese Population. PLoS ONE, 10, $\mathrm{e} 0116110$.

[24] Baldan, A., Tarr, P., Lee, R. and Edwards, P.A. (2006) ATP-Binding Cassette Transporter G1 and Lipid Homeostasis. Current Opinion in Lipidology, 17, 227-232. https://doi.org/10.1097/01.mol.0000226113.89812.bb

[25] Mauldin, J.P., Srinivasan, S., Mulya, A., et al. (2006) Reduction in ABCG1 in Type 2 Diabetic Mice Increases Macrophage Foam Cell Formation. Biological Chemistry, 281, 21216-21224. https://doi.org/10.1074/jbc.M510952200

[26] Barbarossa, G., Renzi, A., D’Erasmo, L., et al. (2014) The Relation between Glycemic Control and HDL-C in Type 2 Diabetes: A Preliminary Step Forward? Diabetes Research and Clinical Practice, 104, 26-28. https://doi.org/10.1016/j.diabres.2013.12.061

[27] Uehara, Y., Engel, T., Li, Z., et al. (2002) Polyunsaturated Fatty Acids and Acetoacetate Downregulate the Expression of the ATP-Binding Cassette Transporter A1. Diabetes, 51, 2922-2928. https://doi.org/10.2337/diabetes.51.10.2922

[28] Chait, A., Han, C.Y., Oram, J.F., et al. (2005) Thematic Review Series: The Immune System and Atherogenesis. Lipoprotein-Associated Inflammatory Proteins: Markers or Mediators of Cardiovascular Disease? The Journal of Lipid Research, 46, 389-403. https://doi.org/10.1194/jlr.R400017-JLR200

[29] Ina, K., Hayashi, T., Araki, A., et al. (2014) Importance of High-Density Lipoprotein Cholesterol Levels in Elderly Diabetic Individuals with Type IIb Dyslipidemia: A 2-Year Survey of Cardiovascular Events. Geriatrics \& Gerontology International, 14, 806-810. https://doi.org/10.1111/ggi.12168

[30] Passarelli, M., Tang, C., McDonald, T.O., et al. (2005) Advanced Glycation End Product Precursorsimpair ABCA1-Dependent Cholesterol Removal from Cells. Diabetes, 54, 2198-2205. https://doi.org/10.2337/diabetes.54.7.2198 
[31] McGillicuddy, F.C., de la LleraMoya, M., Hinkle, C.C., et al. (2009) Inflammation Impairs Reverse Cholesterol Transport in Vivo. Circulation, 119, 1135-1145. https://doi.org/10.1161/CIRCULATIONAHA.108.810721

[32] Sorrentino, S.A., Besler, C., Rohrer, L., et al. (2010) Endothelial-Vasoprotective Effects of High Density Lipoprotein Are Impaired in Patients with Type 2 Diabetes Mellitus But Are Improved after Extended-Release Niacin Therapy. Circulation, 12, 110-122. https://doi.org/10.1161/CIRCULATIONAHA.108.836346

[33] Annema, W., Nijstad, N., Tolle, M., et al. (2010) Myeloperoxidase and Serumamyloid A Contribute to Impaired in Vivo Reverse Cholesterol Transport during the Acute Phase Response But Not Group IIA Secretory Phospholipase A(2). The Journal of Lipid Research, 51, 743-754. https://doi.org/10.1194/jlr.M000323

[34] Zerrad-Saadi, A., Therond, P., Chantepie, S., et al. (2009) HDL3-Mediated Inactivation of LDL Associated Phospholipid Hydroperoxides Is Determined by the Redox Status of Apolipoprotein A-I and HDL Particle Surface Lipidrigidity: Relevance Toinflammation and Atherogenesis. Arteriosclerosis, Thrombosis, and Vascular Biology, 29, 2169-2175. https://doi.org/10.1161/ATVBAHA.109.194555

[35] Briand, F., Prunet-Marcassus, B., Thieblemont, Q., et al. (2014) Raising HDL with CETP Inhibitor Torcetrapib Improves Glucose Homeostasis in Dyslipidemic and Insulin Resistant Hamsters. Atherosclerosis, 233, 359-362.

https://doi.org/10.1016/j.atherosclerosis.2014.01.028

[36] Njajou, O.T., Kanaya, A.M., Holvoet, P., et al. (2009) Association between Oxidized LDL, Obesity and Type 2 Diabetes in a Population-Based Cohort, the Health, Aging and Body Composition Study. Diabetes/Metabolism Research and Reviews, 25, 733-739. https://doi.org/10.1002/dmrr.1011

[37] Liu, F. and Huang, L. (2001) Improving Plasmid DNA-Mediated Liver Gene Transfer by Prolonging Its Retention in the Hepatic Vasculature. The Journal of Gene Medicine, 3, 569-576. https://doi.org/10.1002/jgm.222

[38] Von Eckardstein, A. and Widmann, C. (2014) High-Density Lipoprotein, Beta Cells, and Diabetes. Cardiovascular Research, 103, 384-394. https://doi.org/10.1093/cvr/cvu143

[39] Nobecourt, E., Jacqueminet, S., Hansel, B., et al. (2005) Defective Antioxidative Activity of Small Dense HDL3 Particles in Type 2 Diabetes: Relationship to Elevated Oxidative Stress and Hyperglycaemia. Diabetologia, 48, 529-538.

https://doi.org/10.1007/s00125-004-1655-5

[40] Kon, V., Yang, H. and Fazio, S. (2015) Residual Cardiovascular Risk in Chronic Kidney Disease: Role of High-Density Lipoprotein. Archives of Medical Research, 46, 379-391. https://doi.org/10.1016/j.arcmed.2015.05.009

[41] Mastorikou, M., Mackness, B., Liu, Y., et al. (2008) Glycation of Paraoxonase-1 Inhibits Its Activity and Impairs the Ability of High Density Lipoprotein to Metabolize Membrane Lipid Hydroperoxides. Diabetic Medicine, 25, 1049-1055. https://doi.org/10.1111/j.1464-5491.2008.02546.x

[42] Rozenberg, O., Shih, D.M. and Aviram, M. (2005) Paraoxonase 1 (PON1) Attenuates Macrophage Oxidative Status: Studies in PON1 Transfected Cells and in PON1 Transgenic Mice. Atherosclerosis, 181, 9-18.

https://doi.org/10.1016/j.atherosclerosis.2004.12.030

[43] Feingold, K.R. and Grunfeld, C. (2016) Effect of Inflammation on HDL Structure and Function. Current Opinion in Lipidology, 27, 521-530. https://doi.org/10.1097/MOL.0000000000000333

[44] Rozenberg, O., Rosenblat, M., Coleman, R., et al. (2003) Paraoxonase (PON1) Defi- 
ciency Is Associated with Increased Macrophage Oxidative Stress: Studies in PON1-Knockout Mice. Free Radical Biology \& Medicine, 34, 774-784. https://doi.org/10.1016/S0891-5849(02)01429-6

[45] Modi, K.D., Chandwani, R., Ahmed, I., et al. (2016) Discordance between Lipid Markers Used for Predicting Cardiovascular Risk in Patients with Type 2 Diabetes. Diabetology \& Metabolic Syndrome, 10, 99-102. https://doi.org/10.1016/j.dsx.2015.10.002

[46] Persegol, L., Verges, B., Foissac, M., et al. (2006) Inability of HDL from Type 2 Diabetic Patients to Counteract the Inhibitory Effect of Oxidised LDL on Endothelium-Dependent Vasorelaxation. Diabetologia, 49, 1380-1386. https://doi.org/10.1007/s00125-006-0244-1

[47] Riwanto, M., Rohrer, L., Roschitzki, B., et al. (2013) Altered Activation of Endothelial Anti- and Proapoptotic Pathways by High-Density Lipoprotein from Patients with Coronary Artery Disease: Role of High-Density lipoprotein Proteome Remodeling. Circulation, 127, 891-904.

https://doi.org/10.1161/CIRCULATIONAHA.112.108753

[48] Vita, J.A., Brennan, M.L., Gokce, N., et al. (2004) Serum Myeloperoxidase Levels Independently Predict Endothelial Dysfunction in Humans. Circulation, 110, 1134-1139. https://doi.org/10.1161/01.CIR.0000140262.20831.8F

[49] van der Stoep, M., Korporaal, S.J. and Van Eck, M. (2014) High-Density Lipoprotein as a Modulator of Platelet and Coagulation Responses. Cardiovascular Research, 103, 362-371. https://doi.org/10.1093/cvr/cvu137

[50] Feng, Y., Jacobs, F., Van Craeyveld, E., et al. (2008) Human ApoA-I Transfer Attenuates Transplant Arteriosclerosis via Enhanced Incorporation of Bone Marrow-Derived Endothelial Progenitor Cells. Arteriosclerosis, Thrombosis, and Vascular Biology, 28, 278-283. https://doi.org/10.1161/ATVBAHA.107.158741

[51] Lupattelli, G., Marchesi, S., Roscini, A.R., et al. (2002) Direct Association between High-Density Lipoprotein Cholesterol and Endothelial Function in Hyperlipemia. American Journal of Cardiology, 90, 648-650. https://doi.org/10.1016/S0002-9149(02)02575-4

[52] Ren, X., Chen, Z.A., Zheng, S., et al. (2016) Association between Triglyceride to HDL-C Ratio (TG/HDL-C) and Insulin Resistance in Chinese Patients with Newly Diagnosed Type 2 Diabetes Mellitus. PLoS ONE, 11, e0154345.

https://doi.org/10.1371/journal.pone.0154345

[53] Hanefeld, M., Traylor, L., Gao, L., et al. (2017) The Use of Lipid-Lowering Therapy and Effects of Antihyperglycaemic Therapy on Lipids in Subjects with Type 2 Diabetes with or without Cardiovascular Disease: A Pooled Analysis of Data from Eleven Randomized Trials with Insulin Glargine $100 \mathrm{U} / \mathrm{ml}$. Cardiovascular Diabetology, 16, 66. https://doi.org/10.1186/s12933-017-0548-0

[54] Quispe, R., Martin, S.S., Jones, S.R., et al. (2016) Triglycerides to High-Density Lipoprotein-Cholesterol Ratio, Glycemic Control and Cardiovascular Risk in Obese Patients with Type 2 Diabetes. Current Opinion in Endocrinology, Diabetes and Obesity, 23, 150-156. https://doi.org/10.1097/MED.0000000000000241

[55] Oak, J.H. and Cai, H. (2007) Attenuation of Angiotensin II Signaling Recouplesenos and Inhibits Nonendothelial NOX Activity in Diabetic Mice. Diabetes, 56, 118-126. https://doi.org/10.2337/db06-0288

[56] Kong, D., Melo, L.G., Mangi, A.A., et al. (2004) Enhanced Inhibition of Neointimal Hyperplasia by Genetically Engineered Endothelial Progenitor Cells. Circulation, 109, 1769-1775. https://doi.org/10.1161/01.CIR.0000121732.85572.6F 
[57] Loomans, C.J., de Koning, E.J., Staal, F.J., et al. (2004) Endothelial Progenitor Cell Dysfunction: A Novel Concept in the Pathogenesis of Vascular Complications of Type 1 Diabetes. Diabetes, 53, 195-199. https://doi.org/10.2337/diabetes.53.1.195

[58] Sharif, S., van der Graaf, Y., Nathoe, H.M., et al. (2016) HDL Cholesterol as a Residual Risk Factor for Vascular Events and All-Cause Mortality in Patients with Type 2 Diabetes. Diabetes Care, 39, 1424-1430. https://doi.org/10.2337/dc16-0155

[59] Van Linthout, S., Riad, A., Dhayat, N., et al. (2007) Anti-Inflammatory Effects of Atorvastatin Improve Left Ventricular Function in Experimental Diabetic Cardiomyopathy. Diabetologia, 50, 1977-1986.

https://doi.org/10.1007/s00125-007-0719-8

[60] Vaziri, N.D. (2016) HDL Abnormalities in Nephrotic Syndrome and Chronic Kidney Disease. Nature Reviews Nephrology, 12, 37-47.

https://doi.org/10.1038/nrneph.2015.180

[61] Prufer, N., Kleuser, B. and van der Giet, M. (2015) The Role of Serum Amyloid A and Sphingosine-1-Phosphate on High-Density Lipoprotein Functionality. Biological Chemistry, 396, 573-583. https://doi.org/10.1515/hsz-2014-0192

[62] Nishikawa, T., Edelstein, D., Du, X.L., et al. (2000) Normalizing Mitochondrial Superoxide Production Blocks Three Pathways of Hyperglycaemic Damage. Nature, 404, 787-790. https://doi.org/10.1038/35008121

[63] Westermann, D., Rutschow, S., Van Linthout, S., et al. (2006) Inhibition of p38 Mitogen-Activated Protein Kinase Attenuates Left Ventricular Dysfunction by $\mathrm{Me}$ diating Pro-Inflammatory Cardiac Cytokine Levels in a Mouse Model of Diabetes Mellitus. Diabetologia, 49, 2507-2513. https://doi.org/10.1007/s00125-006-0385-2

[64] Dewald, O., Frangogiannis, N.G., Zoerlein, M., et al. (2003) Development of Murine Is Chemic Cardiomyopathy Is Associated with a Transient Inflammatory Reaction and Depends on Reactive Oxygen Species. Proceedings of the National Academy of Sciences of the United States of America, 100, 2700-2705. https://doi.org/10.1073/pnas.0438035100

[65] Shen, X., Zheng, S., Metreveli, N.S., et al. (2006) Protection of Cardiac Mitochondria by Overexpression of MnSOD Reduces Diabetic Cardiomyopathy. Diabetes, 55, 798-805. https://doi.org/10.2337/diabetes.55.03.06.db05-1039

[66] Suzuki, K., Murtuza, B., Sammut, I.A., et al. (2002) Heat Shock Protein 72 Enhances Manganese Superoxide Dismutase Activity during Myocardial Is Chemia Reperfusion Injury, Associated with Mitochondrial Protection and Apoptosis Reduction. Circulation, 106, 1270-1276. 